Ann. Zootech., I962, 11 (I), 53-59.

\title{
INFLUENCES DE FACTEURS EXTERNES SUR LE DÉCLENCHEMENT DE LA PUBERTÉ CHEZ LA TRUIE
}

\author{
F. DU MESNIL DU BUISSON et J. P. SIGNORET \\ avec la collaboration technique de J. Gautier \\ Station de Recherches de Physiologie animale, \\ Centre national de Recherches zootechniques, Jouy-en-Josas (Seine-et-Oise).
}

SOMMAIRE

1043 jeunes truies de type Large White, achetées à un poids voisin de $90 \mathrm{~kg}$, ont été soumises deux fois par jour, au contrôle de l'œestrus par un verrat boute-en-train.

A partir de l'arrivée à la porcherie expérimentale, on constate une fréquence très importante des premiers œestrus les $4^{\mathrm{e}}, 5^{\mathrm{e}}$, et $6^{\mathrm{e}}$ jours. 277 truies soit $26,5 \mathrm{p}$. Ioo ont leur première chaleur durant un de ces trois jours, alors qu'on en compte seulement 67 et $12 \mathrm{I}$ durant les trois jours précédents et les trois jours suivants.

N'ayant pu connaître l'état sexuel des truies expérimentales au moment de leur entrée à la porcherie, nous avons examiné à l'abattoir un lot d'animaux comparables. Cet examen a permis de constater qu'à ce poids, les $3 / 4$ des truies Large White sont impubères, mais que, parmi celles-ci, environ $\mathrm{I} / 3$ présentent une nette stimulation folliculaire.

On peut supposer que, plus particulièrement chez ces derniers animaux, une stimulation externe provoque la maturation des follicules conduisant à l'cestrus avec un délai de 4 à 6 jours.

Cette stimulation pourrait être due snit au transport et au changement de milieu, soit à la présentation biquotidienne au verrat.

L'apparition de la puberté chez la Truie a été étudiée en détail par de nombreux auteurs : Wiggins, CASIDA et Grummer (I950), Robertson et al. (I95I a et b), WARNick et al. (I95I), Burger (I952), Christian et Nofziger (I952), Seif et al. (r955), Foote et al. (r956), Ito, Kudo et Niwa (I958), GossetT' et SorENSEN (I959), HAINES, WARNICK et WALLACE (I959) ont notamment précisé l'influence de l'âge, du poids, de la race, du taux de consanguinité, de la saison, des conditions d'alimentation et d'élevage.

Sur I 043 truies entretenues pour des études physiologiques, nous avons pu mettre en évidence un groupage surprenant des premiers œestrus à la suite d'influences externes. Ces observations sont exposées et analysées dans la présente étude. 


\section{MATÉRIEL ET MÉTHODES}

Les animaux observés sont de jeunes truies, de race Large White pour la quasi-totalité, achetées à un poids moyen voisin de $90 \mathrm{~kg}$.

Les conditions d'entretien et d'alimentation sont invariables au cours de l'année et sont restées les mêmes pendant les six années d'observation ( 1955 à $196 \mathrm{I}$ ).

La détection de l'état d'o'strus est faite en présentant chaque jour, matin et soir, tous les animaux par lots de 5 ou 6 à un verrat.

690 jeunes truies ont été pesées le jour de leur arrivée. 353 autres n'ont été pesées que plusieurs semaines après et leur poids n'a pas été retenu. Les truies n'étant pas venues en cycle à l'âge d'un in ont été abattues et ne figurent pas dans cette étude.

Enfin, à l'abattoir, nous avons examiné les tractus génitaux de 858 jeunes truies, de poids et de développement comparables aux précédentes afin de déterminer l'état sexuel des animaus de ce type (1).

\section{RÉSULTATS}

L'intervalle séparant l'arrivée à la porcherie du premier astrus, varie de o à I 27 jours. Pour 89,3 p. Ioo des I 043 truies étudiées il est inférieur à 50 jours, la répartition journalière en est présentée dans la figure $I$.

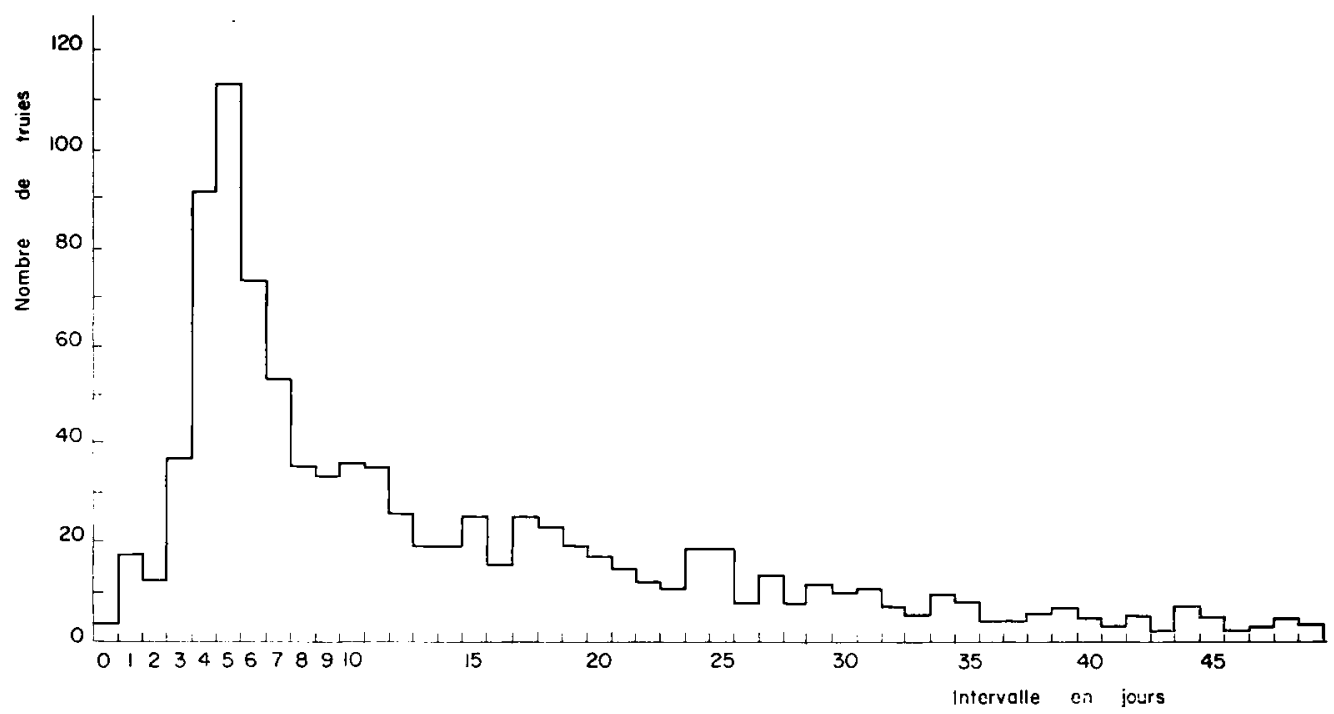

Fis. I. - Intervalle entre l'arrivie à la porcherie et le dibui cel' l'astrus

Que les jeunes truies que nous achetons soient pubères ou non, on devrait normalement avoir une répartition journalière uniforme des premiers œestrus contrôlés ; ceux des truies déjà en cycle apparaissant dans les 2 I premiers jours, de même que ceux des autres truies qui normalement atteignent leur puberté à ce moment.

Or, on constate au contraire une pointe très importante sur trois jours seulement, les $4^{\mathrm{e}}, 5^{\mathrm{e}}$ et $6^{\mathrm{e}}$ jours après l'arrivée des animaux à la porcherie : 277 truies ont le début de leurs chaleurs durant un de ces trois jours ; alors qu'on en compte seulement 67 et I 2 I durant les trois jours précédents et les trois suivants (tableau I).

(I) Nous remercions les Établissements GEO grâce auxquels nous arons pu réaliser ces obscrvations. 
TABLEAU I

Intervalle entre l'arrivée et le premier jour de l'astrus

\begin{tabular}{|c|c|c|c|c|c|c|c|c|c|}
\hline $\begin{array}{c}\text { Intervalle } \\
\text { Poids } \\
\downarrow \\
\end{array}$ & $0 \grave{a} 3 \mathrm{j}$ & $4 \grave{a} 6 j$ & $7 \grave{a} 9 \mathrm{j}$ & $10 \stackrel{a}{12 j}$ & $13 \mathrm{à} 15 \mathrm{j}$ & 16 à $18 \mathrm{j}$ & $19 \mathrm{a} 21 \mathrm{j}$ & $22 \mathrm{j}$ et + & Total \\
\hline $60-69 \mathrm{~kg}$ & 0 & 1 & 0 & 0 & 1 & 0 & 0 & 17 & 19 \\
\hline $70-79 \mathrm{~kg}$ & 1 & 19 & 5 & 5 & 2 & 4 & 3 & 55 & 94 \\
\hline $80-89 \mathrm{~kg}$ & 9 & 47 & 31 & 21 & 12 & 13 & 5 & 62 & 200 \\
\hline $90-99 \mathrm{~kg}$ & 16 & 70 & 24 & 17 & 13 & 14 & 7 & 59 & 220 \\
\hline $100-109 \mathrm{~kg}$ & 10 & 41 & 12 & 9 & 10 & 8 & 9 & 22 & 121 \\
\hline $110-120 \mathrm{~kg}$ & 3 & 9 & 4 & 3 & 4 & 3 & 2 & 8 & 36 \\
\hline $\begin{array}{l}\text { Toutes truies } \\
\text { depoids conrus }\end{array}$ & 39 & 187 & 76 & 55 & 42 & 42 & 26 & 223 & 690 \\
\hline Toutes truies & 67 & 277 & 121 & 97 & 63 & 63 & 52 & 303 & 10,3 \\
\hline
\end{tabular}

D'autre part le pourcentage de truies qui viennent en œstrus pendant cette période de 3 jours est d'autant plus importante que les truies sont plus lourdes, comme le montre la figure 2 . Il passe de $20 \mathrm{p}$. Ioo pour les truies de 70 à $79 \mathrm{~kg}$ à

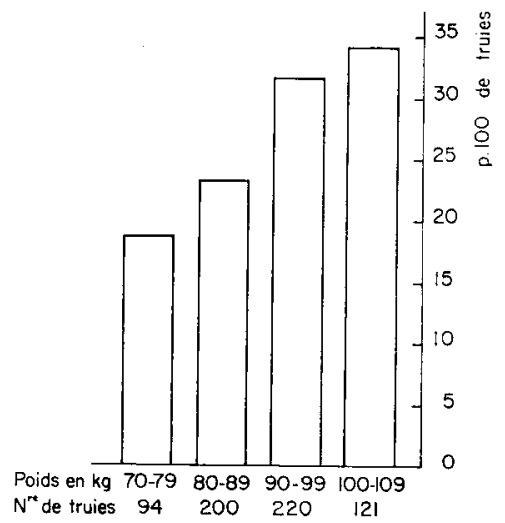

FIG. 2. - Évolution du nombre de truies venant en astrus les $4,5,6 \mathrm{e}$ jours après leur arrivée en fonction de leur poids

plus de $30 \mathrm{p}$. Ioo pour celles d'un poids compris entre 90 et roo $\mathrm{kg}$ (variation significative $\left.\mathrm{X}^{2}=\mathrm{I} 0,883 ; \mathrm{P}<0,02\right)$.

Il était intéressant de connaître l'état sexuel des animaux à leur arrivée, pour savoir si le groupage observé peut être dû à un déclenchement simultané de la puberté ou à une remise en phas a des cycles d'animaux déjà pubères. Seull l'examen direct du 
tractus génital aurait pu nous fournir ces renseignements. Or, cet examen n'a pas pu être effectué sur nos animaux expérimentaux. Nous avons donc examiné à l'abattoir utérus et ovaires d'un lot de 858 truies comparables aux nôtres. 265 (soit 28,4 p. Ioo seulement furent trouvées pubères. Or, parmi les impubères, un nombre relativement élevé d'animaux (2I2 soit 22,8 p. IOO) présentait une nette stimulation folliculaire. (tableau 2).

TABIEAU 2

Résultats des examens des tractus génitaux de Truies de 80 à I $10 \mathrm{~kg}$ à l'abaltoir

\begin{tabular}{|c|c|c|c|c|}
\hline Catégorie & Ovaire & leterus & Nbre de Truies & Pourcentage \\
\hline Infantiles & $\begin{array}{l}\text { Aucun follicule de plus re } \\
5 \mathrm{~mm} \text {; en général au moins } \\
20 \text { follicules rle } 2 \text { à } 3 \mathrm{~mm} \text {. }\end{array}$ & $\begin{array}{l}\text { Fin, ccurt, de couleur blan- } \\
\text { châtre: joids moyen } \\
30 \mathrm{~g} .\end{array}$ & 92 & 9,9 \\
\hline Inpubères & id. & $\begin{array}{l}\text { Plus déveloplé, phus prais, } \\
\text { roloration rose; poids } \\
\text { moyen } 60 \mathrm{~g} \text {. }\end{array}$ & 289 & 31,1 \\
\hline "Prépubères" & $\begin{array}{l}\text { à } 15 \text { follicules de } 5-8 \text { mm, } \\
\text { tendus, non mûrs, peu de } \\
\text { follicules de } 2-3 \mathrm{~mm} \text {. }\end{array}$ & $\begin{array}{l}\text { Térus en géuéral déve- } \\
\text { lopué, ćpais, rose violacé. } \\
\text { foicls de } 100 \text { à } 260 \mathrm{~g} \text {. }\end{array}$ & 212 & 22,8 \\
\hline fre ()vulation & $\begin{array}{l}\text { P'oints d'ovulation datant de } \\
\text { moins de } 2 \text { ' } \mathrm{h} \text { pas de corps } \\
\text { jaunes cn régression. }\end{array}$ & $\begin{array}{l}\text { Córus épais, rose violace ; } \\
\text { poids de } 250 \text { a } 300 \mathrm{k} \text {. }\end{array}$ & 36 & 3,8 \\
\hline 1er Cycle & $\begin{array}{l}\text { Corps james cycliques sans } \\
\text { corps jaunes en régression. }\end{array}$ & $\mathrm{idl}$. & 96 & 10,3 \\
\hline Cycle ultérieur & $\begin{array}{l}\text { Corps jannes cycliques ou folli- } \\
\text { cules de grande taille, avet: } \\
\text { corps jaunes en régression. }\end{array}$ & irl. & $13:$ & 11,3 \\
\hline & - & Total ... & 858 & 100 \\
\hline
\end{tabular}

Ainsi nous sommes amenés à penser que la majorité de nos truies sont impubères au moment où nous les recevons. De plus, puisque nous n'élevons pas d'animaux dans notre porcherie, nous n'avons pas put utiliser comme témoins les truies non transportées et nous ne pouvons que nous référer aux études d'autres auteurs pour affirmer que la puberté s'établit normalement chez les truies d'une façon très progressive entre I80 et 260 jours (BURGER, I952).

\section{DISCUSSION}

Deux hypothèses peuvent être émises quant au mécanisme de ce groupage. Action du "stress" (transport ou changement de milieu).

Les animaux subissent en effet un choc important; changement complet des conditions d'environnement, de logement et de nourriture, transport en camion, souvent sans ménagements, mise en lots avec des animaux inconnus, etc. Dans d'autres espèces, certains auteurs ont montré l'action de divers " stress " sur la sécrétion d'hormones gonadotropes. C'est ainsi que MANDL et ZUCKERMAN (I952) observent une puberté précoce chez de jeunes rattes soumises à un refroidissement; 
cependant des manipulations brutales des animaux ne produisent rien de tel. ZONDEK et TAMARI (I960) notent chez les rattes et les lapines une très forte augmentation du poids des ovaires et des corps jaunes et la présence de follicules hémorragiques à la suite d'une forte stimulation sonore.

SAI-HALASZ (I96I) constate une nette augmentation du taux de gonadotrophines dans le sérum sanguin et du poids des ovaires chez des rattes soumises à des chocs électriques lors de l'alimentation.

On peut donc supposer que les chocs divers subis par les jeunes truies lors du transport et de la mise en lots, pourraient provoquer une décharge d'hormones hypophysaires susceptible de déclencher l'oestrus avec un délai de 4 à 6 jours.

\section{Action de la présentation au mâle.}

Un des faits nouveaux liés à l'arrivée à la porcherie expérimentale est la présentation deux fois par jour de toutes les truies au mâle. En effet, dans les conditions d'élevage normales des jeunes truies, celles-ci sont élevées soit entre femelles, soit, ke plus souvent, avec des mâles castrés bien avant la puberté. Or, plusieurs auteurs ont mis en évidence une action des stimuli olfactifs du mâle sur la physiologie de la reproduction de la femelle. Une revue de ces actions a été récemment publiée par PARKES et BRUCE (I $66 \mathrm{I}$ ).

WhITTEN (I956-I957-I958) observe un groupage des accouplements (46 p. IOO des Souris) 3 jours après la réunion du mâle et de la femelle. Or, si les femelles sont précédemment élevées à proximité d'un mâle, séparé d'elles par un grillage, aucun effet de ce genre n'apparaît. L'auteur conclut à une action des stimuli olfactifs. LAMOND (I959) confirme ces résultats.

De même WATSON et RADFORT (I960) attribuent à des stimuli olfactifs l'influence du bélier dans le déclenchement des cycles des brebis à la fin de l'anœestrus saisonnier, influence signalée par de nombreux auteurs chez la Brebis, TRETJAKov (I949), Srnclair (i950), Schinckei, (I954), Granger (I955), Smith et al. (I958) et chez la Chèvre, Shelton (I960).

Enfin à un autre stade de la reproduction, des stimuli olfactifs semblent agir sur la balance endocrine ainsi que le montrent les résultats obtenus sur le blocage de l'implantation chez les souris par BRUCE (I959, I960 $a$ et $b$, I96 I), BRUCE et PARRo'T'T (I960), Bruce et PARKES (rg6o $a$ et $b$, I96r).

Ainsi dans diverses espèces, il a été démontré que des "stress " variés aussi bien que les stimuli venant du mâle sont capables d'agir sur le fonctionnement utéro-ovarien. Dans ce cas, une action ne serait possible que chez des animaux ayant atteint un stade de développement suffisant. En ce qui concerne la Truie, nous avions été frappés depuis longtemps par la fréquence des tractus génitaux se trouvant à un stade que l'on pourrait appeler " prépubère " chez des truies de 70 à roo $\mathrm{kg}$.

Ce stade est caractérisé d'une part, par un développement de l'utérus assez voisin de celui des jeunes truies pubères, d'autre part, par la présence sur l'ovaire d'un nombre réduit (moins de ro par ovaire) de follicules dont le diamètre dépasse $5 \mathrm{~mm}$. Contrairement aux ovaires de truies impubères, ils ne présentent que peu de follicules de 2 à $3 \mathrm{~mm}$. Mais les follicules sont également bien différents de ceux des truies en ostrus. Ils sont peu saillants, peu colorés et semblent tendus, 
contrairement aux follicules avant l'ovulation qui sont souvent saillants et peu turgescents, et dont la surface rose est finement vascularisée.

L'état des ovaires " prépubères » fait penser à un pro-œestrus prolongé où la majorité des follicules auraient déjà subi leur involution tandis que la croissance de quelques-uns d'entre eux se fait très lentement.

Et on peut supposer que chez certains de ces animaux une stimulation externe provoque une brusque décharge d'hormone gonadotrope qui accélère la croissance des follicules et provoque leur maturation.

Le délai de 4 à 6 jours que nous observons coïncide avec la durée de la phase follicalaire telle que l'ont définie Robinsos et NALBANDov (I95I). Ce même délai correspond à la période minimum pour qu'une suralimentation agisse sur le taux d'ovulation (Zimmerman et al., ig60).

Certes nous ne pouvons pas exclure l'hypothèse d'un groupage des œestrus chez les truies déjà pubètes, par un mécanisme neuro-endocrinien qui inhiberait le fonctionnement des corps jaunes et raccourcirait certains cycles. Mais il nous semble plus logique de supposer l'existence d'un état instable de " prépuberté " chez la Truie ; à ce stade, des influences externes agissant vraisemblablement par voie nerveuse sur la balance endocrine pourraient déclencher la phase de croissance folliculaire conduisant à l'oestrus.

Recu en jamier 1962

\section{SUMMARY}

INFLUENG OF EXTERNAL FACIORS ON THE ONSET OF PLBERTY IN THE GILT.

1043 young large White gilts, bought at a weight of about go $\mathrm{kg}$, were submitted twice a day to a control of the a'strus by a teaser boar.

From the time of arrival at the experimental piggerv, al very important frequency of the first westrus on the 4 th, 5 th and 6 th days is noted. 277 gilts, that is to say $26,5 \mathrm{p}$. 10o, have their first heat during one of these three days, whilst only 67 are counted on the three preceeding days and I 2 I on the three following days.

As it was not possible to know the sexual state of the experimental gilts at the time of their arrival at the piggery, a comparable group of animals were examined at the slaughter-house.This exanination made it possible to establish that, at 1 his weight, $3 / 4$ of the Large White gilts are prepubeeral, but that out of these about $1 / 3$ shows a definite follicular stimulation.

It may be supposed particularly, with the latter animals, that an external stimulation prorokes the maturation of the follicles leading to the cestrus with a delay of 4 to 6 days.

This stimulation might be due to either the transport and change of surroundings, or the twice daily presentation to the boar.

\section{RÉFÉRENCES BIBLIOGRAPHIQUES}

I3RUCE II. M., I959. An exteroceptive block to pregnancy in the House. Nilure, 184, 105.

BRUCE H. M., rofo a. A block to prennancy in the Mouse caused by proxinity of strange males. J. Reprod. liertil., 1, 96- Iо 3 .

BRvCE. H. M., Ig60 b. Further observations on premancy block in Mice caused by the proximity of strange males (Proc.) J. Reprol. Fertil., 1, 31 1-312.

BRECE H. I., Igor. Time relations in the pregnancy block induced in Hice by strange males. J. Reprod. Fertil., 2, I $38-1+2$.

Bruce H. H., Parkes A. S., igho a. Hormonal factors in exteroceptive block to pregnancy in Xice. $J$. Endacrin., 20, XXIX-XXX.

l3ktee H. M., Pakkls A.S., ig6o b. The effect of concument lactation on the olfactory block to presnancy in the House. J. Entorin., 22, vi-viv. 
Brice II. M., Parkes A. S., rgor. An olfactory block to implantation in Mice (Lroc.) J. Reprod. Ferth, 2, 195 .

Bruce II. M., PARrotT O. M. V., ig60. Role of olfactory sense in pregnancy block by st range males. Science, $131,1526$.

Burger I. F., I952. Sex physiology of P'ig. Onderstepoort J. Vet. Re's. suppl., 2, sept 1952, 41-52.

Cintstian R. E., Nofzicter J. C., r952. Puberty and other reproductive phenomena in gilts as affected by plane of nutrition. J. Amim. Sci., 11, 780 .

Foote W. (., Waldorf I), I', Chapman A. B., Self II. L., Grimer R. H., Casida L. F., i956. Age at puberty of gilts produced by different systems of mating. I mim. J. Sii., 15, $959+609$.

Gossett J. W., Sorensen A. II. jr, 1959. The effect of two levels of energy and seasons on reproductive phenomena of gilts. J. Anim. Sci., 18,40-47.

Granger W., i955. Influence of ram on oestrus in ewes. Aust. Vel. /., 31, i 38-139.

Haixes ( $\therefore$ E., Warnick A. (., Wablace II. I)., I959). The effect of two levels of energy intake on reproductive phenomena in duroc jersey gilts. $J$. Imim. Sci, 18, 34-354.

Ito S.. Kivo A., Niwa T., 1958. Studies on the normal oestrus in swine with special reference to proper time for service. In colloque sur la Reproduction et l'Insémination artificielle du I'orc. Inn. Zootech. suppl. I05-107.

LAMONI, D. R., I 959. Effect of stimulation derived from other animals of the same species on oestrous cycle in mice. J. Endocrin., 18, $343-349$.

Maxid. A. M., Zuckermass S., ig52. Factors influencing the onset of puberty in albino rats. J. Endocrin. $8,357 \cdot 364$.

Parkes A.S., Brece H. Il., ig6r. Olfactory stimuli in manmalian reproduction. Science, 134, 1046-10 54 .

Robertson (i.L.. Casion I. J., Grumer R. II., ('hapmax .1. 1., ro5. Some fecding and management factors affecting age at puberty and related phenomena in chester white and poland china crilts. J. Inim. Sci., 10, 647-656.

Robinson (i. E. jr, Nalbanioy A. V., i95I. Changes in the hormone content of swine pituitaries during the estrual cycle. J. Anim. Sit., 10, $469-478$.

SAI-Halasz A., I96r. Gonadostimuling effect of experimental neurosis. In proc. 3rd World congr. Ferlil.

Steril. Internat. Congr. Séries n" 3.3. Excerpta Medical Fundation Amsterdam, London, Xew-York, 995-997.

Schisckes. I'. (i., I954. The effect of the presence of the ram on the ovarian activity of the ewe. Aust. I. Agric. Res., 5, 464-469.

Self II. L., Grummer R. H., CASIDA L. Ji., I95.5. The effect of various sequences of full and limited feeding on the reproductive phenomena in chester white and poland china gilts. $f$. Anim. Sci., 14, 573-592.

SHELToN M., I96o. Influence of the presence of a male goat on the initiation of oestrous cycling and ovulation of angora does. J. Inim. Sci., 19, 368-375.

Sinclatr A. N., 1950. A note on the effect of the presence of rams on the incidence of oestrus in maiden merino ewes during spring mating. tust. Vet. J., 26, 3i-30.

SMmir I. J., McLaren J. B., (Don J. A., MH.L.er H. ,I958. Influence of the use of a sterile teaser mam prior to breeding on subsecuucnt fertility of ewes. I. A imim. Sci., 17, 1231.

TREtjakov N. N., I949. Le róle de facteurs externes sur le cycle sexuel de la Brelbis (en russe). Dohl. . Ikad. Nauk.S.S.S.R., 64, $599-602$.

Warnick A. C., Wiggins (. L., Casma L. li., cirumuer R. H., Chapmax A. B., Ig5. Variation in puberty phenomena in inbred gilts. J. Anim. Sci., 10, $479-49.3$.

Witson R. II., RADFord II. M., Ig6o. The influence of rams on onset of oestrus in Ierino ewes in the spring. Aust. J. Agric. Res., 11, 65-7I.

Winttes W. K., I 956. Modifications of oestrus cycle of the mouse by extemal stimulation associated with the male. J. Endocrin., 13, 399-404.

Whitten W. K., 1957. Eiffect of exteroceptive fartors on the oestrous cycle of nice. Nalure, 180, r 436.

WhitTex W. K., 1958. Modifications of oestrous cycle of the mouse by external stimuli associated with the male. Changes in the oestrons cycle determined by vaginal smears. J. Endocrin., 17, $307 \cdot 313$.

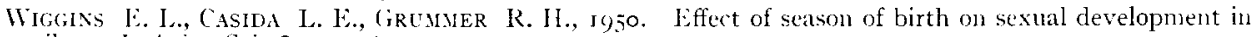
gilts. J. Inim. Sci., 9, 277 -28o.

Zimmermak, 1). R., Spies H. C., Rigor Ji. V., Skll H. L., Casida I.. J., ig6o. liffect of restricted feeding, cross breeding and season of birth on age at puberty in swine. J. Anim. Sci., 19, 687.694.

Zimmerman D. R., Spies II. (i., Self H. L., Casidi I. E., ig6o. Ovulation rate in swine as affected by increased energy intake just prior to ovulation. I. Anm. Sci., 19, 295-301.

ZONDEk R., TaMari I., ig6o. Effect of androgenic stimulation on gental function and reproduction AmerJ. Obst. cinec., 8, 1041-1048 